\title{
Solid-State Detector SPECT Myocardial Perfusion Imaging
}

\author{
Piotr J. Slomka ${ }^{1}$, Robert J.H. Miller ${ }^{1}$, Lien-Hsin $\mathrm{Hu}^{1,2}$, Guido Germano ${ }^{1}$, and Daniel S. Berman ${ }^{1}$ \\ ${ }^{1}$ Department of Imaging, Medicine, and Biomedical Sciences, Cedars-Sinai Medical Center, Los Angeles. California; and \\ ${ }^{2}$ Department of Nuclear Medicine, Taipei Veterans General Hospital, Taipei, Taiwan
}

There has been an evolutionary leap in SPECT imaging with the advent of camera systems that use solid-state crystals and novel collimator designs configured specifically for cardiac imaging. Solid-state SPECT camera systems have facilitated dramatic reductions in both imaging time and radiation dose while maintaining high diagnostic accuracy. These advances are related to simultaneous improvement in photon sensitivity due to the collimator and imaging geometry, as well as image resolution due to the improved energy resolution of the new crystals. Improved photon sensitivity has facilitated fast or low-dose myocardial perfusion imaging (MPI), and early dynamic imaging has emerged as a technique for assessing myocardial blood flow with SPECT. Lastly, general-purpose solid-state camera systems and hybrid SPECT/CT systems have also been developed that may have important clinical roles in cardiac imaging. This review summarizes state-of-the-art solid-state SPECT MPI technology and clinical applications, including emerging techniques for SPECT MPI flow estimation. We also discuss imaging protocols used with the new cameras, potential imaging pitfalls, and the latest data providing large-scale validation of the diagnostic and prognostic value of this new technology.

Key Words: instrumentation; solid-state SPECT; SPECT MPI

J Nucl Med 2019; 60:1194-1204

DOI: 10.2967/jnumed.118.220657

\section{$\mathbf{M}$}

yocardial perfusion imaging (MPI) with SPECT is widely used for the diagnosis and management of patients with known or suspected coronary artery disease, providing crucial information regarding both myocardial perfusion and function. Since being introduced in the 1970s, SPECT camera system designs have undergone several incremental improvements. More recently, however, there has been an evolutionary leap with dedicated cardiac SPECT camera systems that use solid-state crystals and novel collimator designs, such as multipinhole and locally focusing collimators configured specifically for cardiac imaging (1). These innovations have facilitated dramatic reductions in both imaging time and radiation dose while maintaining high diagnostic accuracy. These features are related to simultaneous improvement in sensitivity (5-8 times higher $(2,3)$ due to the collimator and imaging geometry) and image resolution (up to 2 times higher (3) due to the improved energy resolution of the new crystals).

Received Apr. 19, 2019; revision accepted Jun. 18, 2019.

For correspondence or reprints contact: Piotr J. Slomka, Cedars-Sinai Medical Center, 8700 Beverly Blvd., A047N, Los Angeles, CA 90048.

E-mail: slomkap@cshs.org

Published online Aug. 2, 2019.

COPYRIGHT (C) 2019 by the Society of Nuclear Medicine and Molecular Imaging.
Additionally, the physical space requirements for solid-state camera systems have been significantly reduced because photomultiplier tubes are no longer required. New solid-state scanners can also be coupled with diagnostic-quality CT systems suitable for calcium scanning or coronary CT angiography. With the growing clinical experience using solid-state SPECT camera systems, there is also emerging evidence demonstrating comparable or superior diagnostic and prognostic utility.

This review summarizes current solid-state SPECT technology and clinical applications, including emerging techniques for myocardial blood flow (MBF) quantitation. We will also discuss imaging protocols, potential imaging pitfalls, and the validation efforts for this new technology regarding diagnosis and prognosis.

Several technologic advancements are responsible for the improved imaging characteristics of solid-state SPECT camera systems. No single change can be attributed to the significant improvements in both photon sensitivity and spatial resolution realized with these systems. We review these advances within the context of the overall camera system.

\section{SOLID-STATE DETECTORS}

The radiation detectors are made of cadmium-zinc-telluride (CZT) crystals, which are more expensive than $\mathrm{NaI}(\mathrm{Tl})$ crystal detectors but have important imaging advantages. CZT detectors have an improved energy response, which reduces the scatter component of measured data by $30 \%$ (4), and superior intrinsic spatial resolution compared with conventional Anger cameras. Removing the need for photomultiplier tubes has resulted in a more compact detector size, which has facilitated more efficient camera designs with innovative imaging geometries and high-sensitivity collimation. Solid-state detectors can also be combined with scintillation crystals to indirectly detect photons. In these detectors, thalliumdoped cesium iodide $\mathrm{CsI}(\mathrm{Tl})$ scintillation crystals are coupled with photodiodes (instead of photomultipliers) (5). CsI(Tl) has superior spectral light response characteristics compared with $\mathrm{NaI}(\mathrm{Tl})$ when used with silicon photodiodes.

Solid-state detectors are integrated with innovative collimator designs that increase photon sensitivity in the myocardial region. The high-sensitivity characteristics of solid-state SPECT cameras are often mistakenly attributed to the CZT crystal. Although the density of CZT is higher than $\mathrm{NaI}(\mathrm{Tl})\left(5.8\right.$ vs. $\left.3.7 \mathrm{~g} / \mathrm{cm}^{3}\right)$, because of cost considerations CZT detectors are thinner $(\sim 5 \mathrm{~mm})$ than typical $\mathrm{NaI}(\mathrm{Tl})$ crystals $(9.5 \mathrm{~mm})$, such that the intrinsic detection efficiency of these systems is comparable to conventional Anger detectors.

\section{DEDICATED CARDIAC DETECTORS}

CZT SPECT camera systems specifically designed for cardiac use are commercially available from 2 vendors. One system (D-SPECT; 
Spectrum Dynamics) uses pixelated CZT detector arrays. Nine vertical columns are arranged in a $90^{\circ}$ gantry geometry. Each column consists of $1,024 \mathrm{CZT}$ elements $(2.46 \times 2.46 \times 5 \mathrm{~mm}$ thick), arranged in a $16 \times 64$ element array with an overall size of $40 \times 160 \mathrm{~mm}$. Since CZT crystals are expensive, the vendor offers a system with 6 detector columns, creating a trade-off between cost and image quality. The other camera design (Discovery NM 530c; GE Healthcare) features a curved array of 19 CZT pixelated detector arrays coupled to pinhole collimators with geometry optimized for cardiac imaging. Each detector array consists of 4 detectors, each with $246 \mathrm{CZT}$ detector elements $(2.46 \times$ $2.46 \times 5 \mathrm{~mm}$ thick) arranged in $16 \times 16$ elements.

The indirect solid-state detector has been used in traditional 2- or 3-detector configurations (Digirad), allowing for a more compact design than conventional Anger cameras. Each detector head contains an array of $768(6.1 \times 6.1 \times 6 \mathrm{~mm}$ thick $) \mathrm{CsI}(\mathrm{Tl})$ crystals coupled to individual silicon photodiodes that convert the crystal scintillations to electrical pulses, which are then processed digitally.

\section{DEDICATED CARDIAC COLLIMATORS AND GEOMETRIES}

The improved sensitivity of solid-state SPECT cameras is achieved by novel collimator designs and dedicated cardiac imaging configurations. Increasing photon sensitivity is important since a conventional Anger camera detects less than $0.01 \%$ of incoming photons (6). Diagrams of these novel collimator technologies, for cardiac solid-state cameras and a general-purpose solid-state camera, are shown in Figure 1.

The D-SPECT features 1 small, high-sensitivity tungsten collimator for each detector column (as described above). These collimators can be positioned close to the patient's body, which increases the number of photons detected and improves spatial resolution. The collimators have square, parallel holes with dimensions of $2.26 \times 2.26 \mathrm{~mm}$ and wall thickness of $0.2 \mathrm{~mm}$ matched to the dimensions of a single detector element. The hole dimensions are larger than for conventional high-resolution collimators, resulting in higher sensitivity at the expense of image resolution. However, the loss in spatial resolution resulting from this design is more than compensated for by the use of the small, pixelated CZT detectors and software-based resolution recovery applied during image reconstruction (2). During acquisition, each of the 9 detectors is focused on the heart and rotates over $45^{\circ}$, with a rotation time of $3 \mathrm{~s}$. Patients are positioned on a chair rather than on a bed, increasing patient comfort. Patients are typically imaged in both semisupine and upright positions.

The Discovery NM 530/570c camera systems use pinhole collimation. The design includes 19 pinhole collimators with multiple focal depths and 1 hole $(4.75 \mathrm{~mm}$ in diameter) in each collimator. The CZT detectors and collimators do not move,

\section{NOTEWORTHY}

- This review summarizes the technologic advancements in commercially available solid-state camera systems and a few systems still in development.

- Emerging data regarding MBF measurements with solidstate SPECT camera systems are summarized.

- Evidence providing large-scale validation of the diagnostic and prognostic utility of solid-state SPECT camera systems for MPI is outlined. and photons are acquired simultaneously through all the pinholes. Simultaneously acquired pinhole views allow consistent tomographic sampling, reducing motion artifacts. Patients are typically imaged in both supine and prone positions, with their arms placed above their head. Higher sensitivity is achieved by the wider acceptance angle of photons with multipinhole collimation and the cardiocentric acquisition, which limits photon detection to the myocardial region.

\section{SOLID-STATE SPECT/CT SYSTEMS}

MPI can benefit from CT attenuation correction (AC) using either dedicated SPECT/CT systems or separate acquisitions and provides a new opportunity to enhance MPI with additional CT information; for example, by obtaining the coronary calcium score. Initially, only multipinhole solid-state camera systems (GE Healthcare 570c) were offered with a hybrid diagnostic CT configuration; however, there are only a few such systems operating worldwide.

In the last 2 years, general-purpose solid-state SPECT cameras coupled with diagnostic-quality CT scanners-capable of coronary calcium scoring-have been introduced by 2 vendors. Although these systems are designed for general imaging use, they can also be used effectively for MPI. Spectrum Dynamics introduced the Veriton-CT system with 8 CZT modules within each of 12 detector blocks, each with individual detector arms with swivel motion, which allow precise and close 3-dimensional contouring around the patient during imaging. The field-of-view length is $320 \mathrm{~mm}$ and can be focused for single-organ acquisitions or used for whole-torso scanning. The optimized collimator design results in higher volumetric sensitivity $(0.0192 \%)$ than dual-head camera systems with conventional collimators $(<0.01 \%)$. Additionally, with image resolution recovery, this system achieves high spatial resolution $(4.3 \mathrm{~mm})$. Initial clinical studies of the prototype design with a detector design similar to Veriton demonstrate potential improvements in image quality compared with a conventional camera (7). The Veriton-CT system is configured with up to a 128-slice CT camera, allowing both calcium scoring and CT angiography studies. GE Healthcare introduced a general-purpose solid-state camera, the Discovery NM/CT 870 CZT, equipped with conventional collimators. This camera system offers similar photon sensitivity to the conventional Anger system (as the collimation system is the same) but allows better energy resolution and image resolution due to the CZT detectors and has demonstrated improved image quality in early clinical implementation $(7,8)$.

Hybrid SPECT/CT solid-state systems are not yet in widespread clinical use. However, separate noncontrast CT coronary artery calcium scans are increasingly combined with SPECTMPI studies. It has been shown that separately acquired coronary artery calcium scans can be used for AC of SPECT MPI studies (9). The use of separately obtained CT for AC is supported by vendors for routine clinical use $(10,11)$.

\section{RECONSTRUCTION INCLUDING RESOLUTION RECOVERY AND ANATOMIC CONSTRAINTS}

In parallel with improvements in technical aspects of SPECT scanner hardware, enhanced image reconstruction techniques have been implemented. New reconstruction algorithms include modeling of the collimators and detectors, as well as resolution recovery. Most systems compensate for spatial resolution losses using iterative reconstruction. The aggressive resolution recovery is 


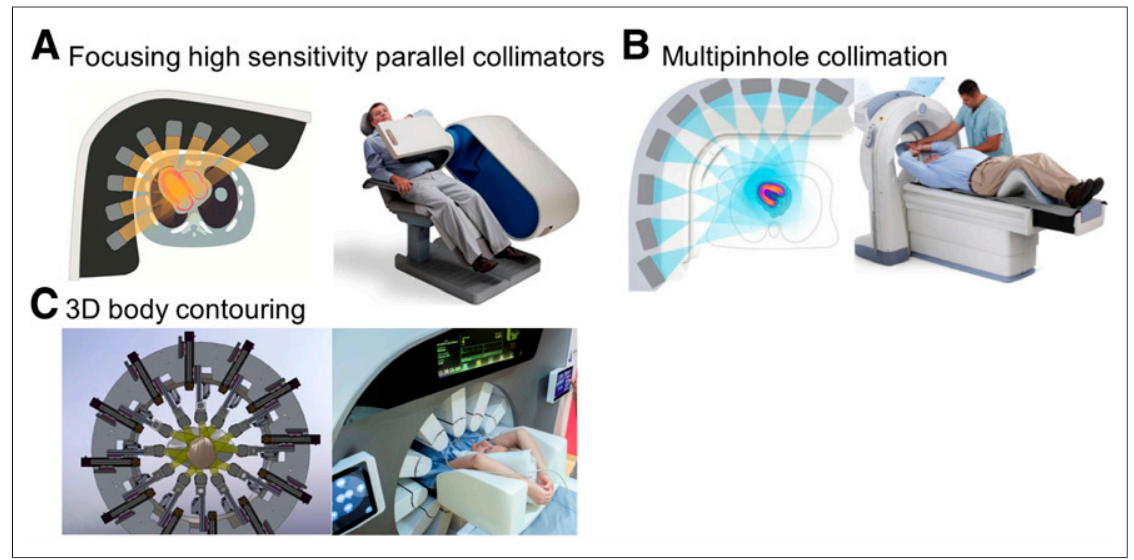

FIGURE 1. Three novel collimator designs used in solid-state camera systems. (A) High-sensitivity parallel collimators, as used in D-SPECT camera system. Collimators are square with parallel holes, each coupled to a single detector. (B) Multipinhole collimator design, as used in Discovery NM 530c camera system. (C) Collimator design of new general-purpose Veriton for 3-dimensional body contouring.

needed to compensate for the effects of high-sensitivity collimation, which reduces spatial resolution by accepting photons from a wider angle. Therefore, software modeling the collimator response during reconstruction is a critical component needed to achieve the simultaneous increase in spatial resolution and count sensitivity with the solid-state SPECT camera systems.

Further improvements in spatial resolution can be achieved using iterative reconstruction with myocardial anatomic priors, which constrain the shape and thickness of myocardial walls to fit within an expected range. This approach can further improve image quality, especially for noisy images. However, if the actual patient anatomy differs significantly from the expected pattern, the convergence of the algorithm may be falsely biased by the assumed constraints. CT scan data can further enhance image reconstruction approaches. With the hybrid SPECT/CT camera systems, the CT can be used during the reconstruction not only for $\mathrm{AC}$ but also as a prior to improve the anatomic constraints.

\section{PERFORMANCE}

The clinical solid-state SPECT systems offer improved sensitivity and spatial resolution compared with conventional Anger cameras. The actual technical performance of solid-state SPECT cameras was compared with conventional dual-head scanners by Imbert at al (3). Additionally, similar results are available from one other vendor. Comparison of count sensitivity (expressed in $\%$ ) with PET are shown in Figure 2. The solid-state SPECT cameras offer up to 8 times higher count sensitivity and more than double the effective image resolution of the conventional Anger cameras. Imaging times with conventional doses of radioactivity are in the range of 2-3 min for systems with dedicated solid-state detectors $(12,13)$, compared with 15 min on systems with a standard collimator. The count sensitivities for dedicated cardiac CZT systems can approach those of 2-dimensional PET systems, which to date have been used in most of the PET-MPI literature.

\section{CURRENT CLINICAL USE}

It is estimated that over 820 solid-state dedicated cardiac CZT SPECT camera systems are currently in clinical use (Yoel Zilbertein and Gil Kovalski, oral communication, March 1, 2019). The improved technical performance of solid-state SPECT camera systems was initially used to dramatically reduce imaging time, improving patient comfort and minimizing motion artifacts. However, clinical protocols with these systems can also use very low-dose radiopharmaceutical injections (148-222 MBq [4-6 mCi] of the ${ }^{99 \mathrm{~m}} \mathrm{Tc}$ perfusion agents) $(14-16)$ with standard imaging times. When using stressonly scans with 10- to 12-min acquisitions, effective radiation doses under $1 \mathrm{mSv}$ are achieved $(17,18)$. By comparison, the average annual background radiation exposure in the United States is approximately $3.1 \mathrm{mSv}(19)$.

\section{2-POSITION IMAGING: UPRIGHT/ SUPINE OR SUPINE/PRONE}

Most solid-state SPECT cameras in clinical use are not in hybrid SPECT/CT configurations and therefore do not offer CT-based AC. At the same time, very low-dose protocols with reduced image counts challenge the accuracy of visual interpretation, especially in the presence of attenuation artifacts. To address this issue, most clinical protocols use 2 sequential scans performed in different patient positions (supine/upright or supine/prone). Since soft tissue shifts in different patient positions, comparing results from 2 positions can allow differentiation of true perfusion defects from artifacts (20-22). Two-position protocols add only modestly to the total time of single-view protocols with high-sensitivity scanners, with no added radiation exposure.

\section{LOW-DOSE PROTOCOLS}

The technical advancements of solid-state SPECT cameras can be used to dramatically reduce radiation exposure during a patient's scan. The list-mode capabilities of SPECT equipment can be used to

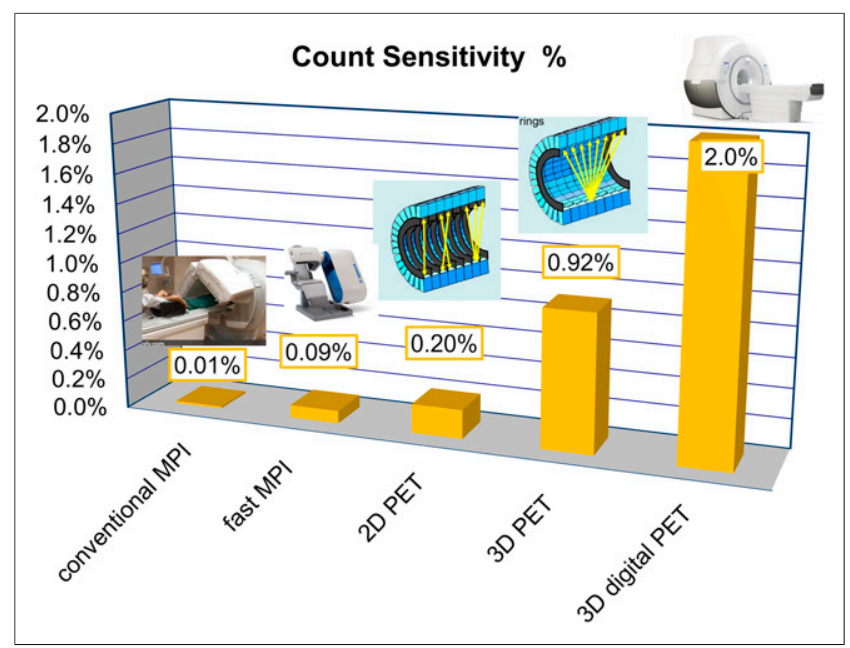

FIGURE 2. Count sensitivity expressed as percentage of incoming photons for several nuclear imaging systems. Solid-state SPECT cameras (fast MPI) have almost 8 times higher count sensitivity than conventional Anger cameras. Therefore, count sensitivity is more similar to 2-dimensional PET than to conventional SPECT camera. 
evaluate retrospectively the efficacy of low-dose imaging protocols. One such study simulated reduced radiation protocols in 79 patients, who were imaged with a solid-state scanner for 14 min with 802.9 $\pm 199.8 \mathrm{MBq}(21.7 \pm 5.4 \mathrm{mCi})$ of ${ }^{99 \mathrm{~m}} \mathrm{Tc}$ injected at stress $(18)$. There was no significant difference in quantitative perfusion or functional measurements even with simulated activity corresponding to an effective radiation dose of less than $1 \mathrm{mSv}$, as outlined in Figure 3. The clinical implementation of low-dose protocols was studied in patients who underwent repeat rest scans with split radiation doses. The image quality for very low-dose rest scans (mean effective dose, $1.15 \mathrm{mSv}$ ) was superior to conventional SPECT imaging (mean effective dose, $2.39 \mathrm{mSv}$ ) (23). Einstein et al. reported stress-only imaging in 69 patients with a mean effective radiation dose of $0.99 \mathrm{mSv}$ (24). In a study of 284 patients undergoing SPECT MPI, Sharir et al. demonstrated similar diagnostic accuracy with low-dose imaging compared with standard dose imaging, but with $50 \%$ radiation reduction (25). This was confirmed by another group who used $160-\mathrm{MBq}$ injected doses for rest and stress acquisitions of $15 \mathrm{~min}$ (26). Palyo et al. showed that regional perfusion defect size and functional parameters were similar despite $50 \%$ radiation reductions using a hybrid SPECT/CT system with CT-based AC (27). With more aggressive radiation reduction (75\%), there was a tendency to overestimate perfusion defect size in patients with abnormal results (27). Importantly, to preserve reproducibility between high-count and low-count data, the quantification of low-dose protocols requires normal limits specifically calibrated with low-dose images because of higher variability of low-count data (28).

As noted above, the lowest total radiation exposure with current SPECT MPI $(\sim 1 \mathrm{mSv})$ can be accomplished clinically by performing stress-only imaging with a solid-state camera system. Stress-only MPI can reduce radiation exposure by up to $60 \%$ (29) and also provides a significant reduction in radiation exposure to staff performing the scan (30). Additionally, stress-only protocols dramatically shorten total study times to as low as 30-45 min, including the stress test, improving the patient experience and laboratory efficiency. Since most SPECT MPI scans are normal (31), an ultralow-dose $(1 \mathrm{mSv})$ stress-only scan could potentially be performed in most patients undergoing SPECT MPI with a solid-state camera system. Further research is needed to demonstrate the diagnostic accuracy and prognostic value of this ultralow-dose, stress-only approach.

\section{SIMULTANEOUS DUAL-ISOTOPE MPI}

Solid-state cameras with CZT detectors have superior energy resolution, which allows better separation of photons from different isotopes and improved cross-talk correction (32). In addition, stationary detector systems eliminate projection inconsistencies that confound dual-isotope imaging. One possible clinical application is a combined rest/stress protocol with simultaneous imaging of ${ }^{99 \mathrm{~m}} \mathrm{Tc}$ and ${ }^{201} \mathrm{Tl}$ as proposed for conventional cameras (33), which was not implemented clinically on standard cameras because of technical difficulties and concerns regarding higher radiation with ${ }^{201} \mathrm{Tl}$.

To date, 3 studies have demonstrated the feasibility of simultaneous imaging of ${ }^{201} \mathrm{Tl}$ (stress) and ${ }^{99 \mathrm{~m}} \mathrm{Tc}$ (rest) SPECT MPI with solid-state cameras. One study proposed a single stress/rest 20-min acquisition for the 2 isotopes (34). A separate study demonstrated the feasibility of simultaneous dual-isotope imaging in 24 patients with $80 \mathrm{MBq}$ of ${ }^{201} \mathrm{Tl}$ injected at rest and $250 \mathrm{MBq}$ of ${ }^{99 \mathrm{~m}} \mathrm{Tc}$ sestamibi injected during adenosine infusion (35). For this application, dedicated scatter correction methods are required because triple-energy window corrections overestimate scatter and cross-talk in CZT-based systems (36). These dedicated corrections have been shown to improve defect contrast and myocardium-to-blood-pool ratios compared with triple-energy window corrections (36). Diagnostic performance and image quality of simultaneous dual-isotope MPI with solid-state systems have been demonstrated to be comparable to conventional SPECT with separate stress/rest acquisitions (32).

Although use of dual-isotope MPI with ${ }^{99 \mathrm{~m}} \mathrm{Tc}$ and ${ }^{201} \mathrm{Tl}$ is now discouraged, simultaneous dual-isotope SPECT may become increasingly used for other imaging protocols. As an example, simultaneous dualisotope imaging for cardiac sarcoidosis with ${ }^{99 \mathrm{~m}} \mathrm{Tc}$-pyrophosphate and ${ }^{201} \mathrm{Tl}$ has recently been described, with ${ }^{201} \mathrm{Tl}$ allowing precise definition of myocardial boundaries (37). This dual-isotope approach has importance for dedicated cardiac systems in which the tight gantry and imaging focused on the heart make it is harder to correctly position the ${ }^{99 \mathrm{~m}}$ Tc-pyrophosphate acquisitions. Notably, CT attenuation imaging could play a similar role in hybrid SPECT/CT systems.

Simultaneous dual-isotope imaging can also be used in combined SPECT perfusion/ metabolism protocols. Many novel metabolism agents, either in clinical or research use, only show abnormal portions of the myocardium, which are better understood within the context of perfusion imaging. As an example, ${ }^{123}$ I-betamethyl- $p$-iodophenyl-pentadecanoic acid has diagnostic and prognostic potential after acute myocardial infarction or in patients
FIGURE 3. Patient with abnormal perfusion in low-dose simulation study performed by Nakazato et al. (18). TPD, which is quantitative measure of ischemia, was not significantly different except in simulations representing effective radiation dose $<0.5 \mathrm{mSv}$. (Reprinted from (18).) 
with Takotsubo cardiomyopathy (38). Solid-state cameras have been shown to simultaneously acquire ${ }^{99 m}$ Tc-perfusion-agent and ${ }^{123}$ I-betamethyl- $p$-iodophenyl-pentadecanoic acid images with image quality similar to that achieved with separate acquisitions (39). Simultaneous metaiodobenzylguanidine and ${ }^{99 \mathrm{~m}} \mathrm{Tc}(40)$, or ${ }^{123} \mathrm{I}-$ metaiodobenzylguanidine and ${ }^{201} \mathrm{Tl}$ imaging can also be acquired (41). In phantom studies, ${ }^{123} \mathrm{I}$ and ${ }^{99 \mathrm{~m}} \mathrm{Tc}$ images can be acquired simultaneously with image quality similar to separate acquisitions with 2 commercially available solid-state cameras (42). Simultaneous dual-isotope acquisitions, which are facilitated by the highenergy resolution of CZT crystals, reduce imaging time, ensure precise image coregistration, and reduce artifacts related to patient or camera motion between acquisitions.

\section{NORMAL PERFUSION LIMITS FOR SOLID-STATE CAMERAS}

Solid-state SPECT scanners have significantly different regional count distributions than standard Anger cameras-primarily due to different types of collimation and detector geometry. Early studies of CZT camera systems demonstrated these differences, and dedicated normal limits were subsequently recommended (13), with dedicated normal databases created for the Discovery NM 530c (GE Healthcare) $(22,43)$ and D-SPECT $(13,20)$ camera systems. Importantly - for optimal quantification-separate databases are required for prone and supine acquisitions.

\section{COMBINED QUANTIFICATION FROM 2 POSITIONS}

Physicians use multiple imaging positions to visually differentiate attenuation artifact from perfusion deficits; however, this approach has only more recently been applied to quantitative analysis. Combined quantitative analysis of upright and supine acquisitions on the solid-state D-SPECT scanner has been evaluated (20). Quantification performed jointly from coregistered images obtained in 2 positions offered better identification of attenuation artifacts and reduced false-positive defects (Fig. 4). Compared with a reference of invasive coronary angiography, combined quantitative analysis showed higher diagnostic accuracy than separate upright or supine acquisitions. Similar results have been shown with 2-position imaging in obese patients using the D-SPECT system (21) and for the Discovery NM530c scanner with supine and prone acquisitions (22). Two-position imaging may also detect position-related truncation artifacts, which can occur because of the limited field of view on solid-state SPECT cameras (44). New approaches applying deep learning with combined 2-position imaging on solidstate cameras have demonstrated further improvements (45).

\section{MOTION CORRECTION ON SOLID-STATE CAMERAS}

Respiratory and patient motion can degrade SPECT image quality, with several recent studies investigating techniques to mitigate these effects. Because of improvements in spatial resolution, heart motion has become the dominant motion-related degrading factor in solid-state SPECT imaging. The feasibility of using list-mode data, with registration of consecutive short-time image frames, to detect and correct for respiratory motion has been demonstrated on these new systems (46). Respiratory gating can impact functional cardiac parameters, resulting in reduced volume estimates and differences in regional left ventricular wall motion (47). End-expiratory gating can be used to improve contrast differences between the myocardium and blood pool (48). Respiratoryphase-matched CT attenuation maps may further improve image quality and have been shown to significantly improve interobserver

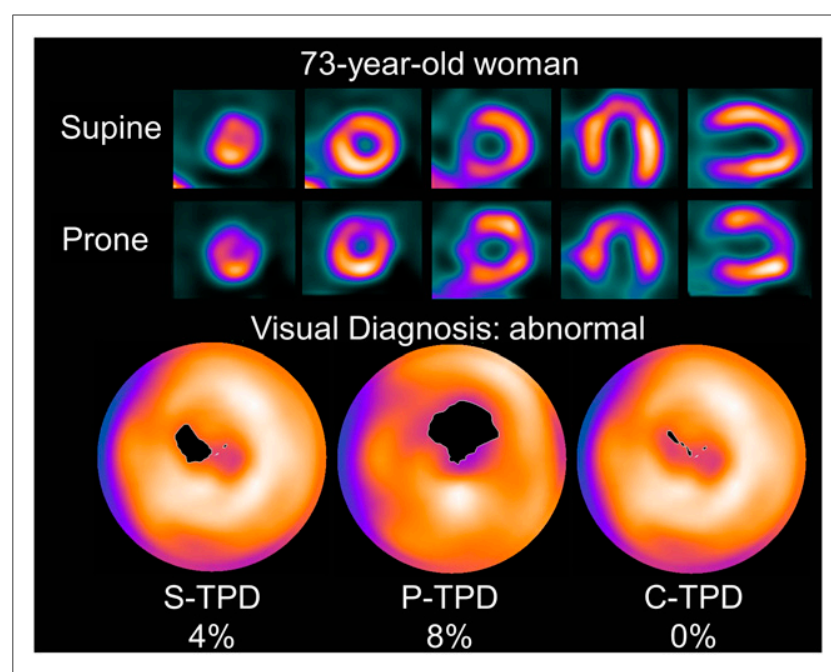

FIGURE 4. Two-position imaging. In this case, combination of supine and prone imaging offers superior visual interpretation, but findings were still interpreted as abnormal. Quantitative perfusion analysis is also improved by integrating supine TPD (S-TPD), and prone TPD (P-TPD) into single, combined measure (C-TPD), which shows no perfusion deficit. Patient had no significant coronary disease on invasive coronary angiography. Case is from REFINE SPECT.

agreement compared with uncorrected images (49). Patient motion can also be corrected using a similar approach to reduce the rate of false-positive perfusion defects (50). Dual respiratory/cardiac gated MPI has been demonstrated in phantoms and patients, showing progressive improvement of the myocardium-to-blood-pool contrast with the application of respiratory and then dual gating (48).

\section{POTENTIAL PITFALLS}

Although the novel designs of dedicated cardiac solid-state SPECT camera systems offer important clinical benefits, there are potential imaging pitfalls that need to be considered. The camera geometry is optimized for cardiac acquisitions, so there is nonuniform count sensitivity in the field of view (51). Therefore, patient positioning is critical and patients with significantly abnormal cardiac anatomy may not be well imaged. Importantly, the typical attenuation defect with supine imaging is more lateral with solid-state camera designs than with conventional cameras (52). For example, because of increased space between the chest wall and cardiac border, obese patients may require repositioning or multiple-position imaging to ensure diagnostic quality (44). In patients with hypertrophic cardiomyopathy, iterative reconstruction algorithms using a heart-shaped model significantly underestimate wall thickness compared with ordered-subsets expectation maximization, which does not use a shape constraint (53). Twoposition protocols may allow for detection of position-related artifacts and potentially address some of these concerns (21).

\section{SPECT MBF}

Absolute MBF and myocardial flow reserve (MFR) provide clinically meaningful data beyond that obtained by assessment of relative perfusion deficit alone. Absolute measurements can identify patients with multivessel coronary artery disease and predict the extent of disease more accurately than relative perfusion quantification or visual interpretation (54). MFR is 


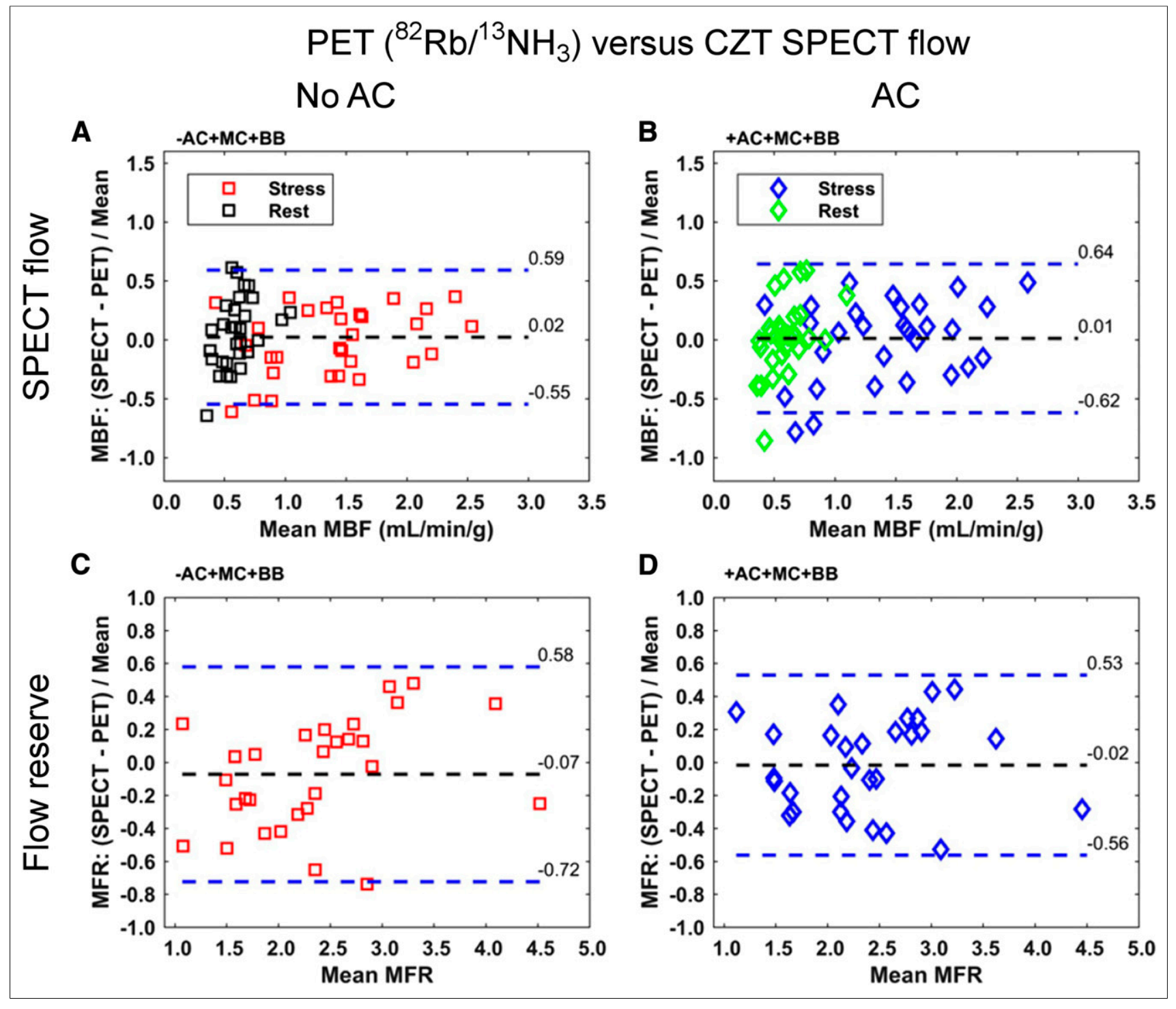

FIGURE 5. SPECT MBF assessments with and without AC compared with PET. SPECT MBF measurements at rest or with stress were not significantly different from PET values, with or without AC. Similar results were obtained with MFR measurements. BB = binding to red blood cells; $\mathrm{MC}=$ motion correction. (Reprinted from (59).)

also a useful prognostic marker, significantly improving risk stratification beyond relative perfusion defects $(55,56)$. Although myocardial flow measurements were initially developed and validated in PET imaging, SPECT cameras may be capable of obtaining similar measurements. The improved count sensitivity related to collimator and camera geometry advances with solid-state SPECT cameras may make them particularly well suited for flow measurements.

Human dynamic SPECT flow studies have been demonstrated on high-efficiency dedicated SPECT, with factor analysis and a 2compartment model used for quantification (57). The clinical feasibility was demonstrated in 95 patients, in whom the data were reconstructed to 3-s frames resulting in 60-70 frames for each stress and rest study. The authors demonstrated that MFR derived from such studies was higher in patients with normal scans (as established by standard relative quantification) and that lower MFR (both regional and global) was associated with a static-imaging stress perfusion defect, age, and smoking. The MFR also showed stepwise reduction related to the severity of the obstructive disease in a subset of 20 patients on whom invasive coronary angiography was performed. To date, at least 7 studies have been performed on humans demonstrating the SPECT MBF measurements with ${ }^{99 \mathrm{~m}} \mathrm{Tc}$ and solid-state cameras (57-63). Importantly, Wells et al. have demonstrated close agreement between SPECT $\mathrm{MBF}$ and MFR measurements, with or without AC, compared with PET measurements (Fig. 5) (59). This finding has important implications, since most solid-state scanners are currently operating without AC capabilities. The clinical protocol for SPECT flow has gained significant attention recently, as the Food and Drug Administration approved dedicated clinical software with both solid-state scanners, with several sites commencing clinical SPECT flow imaging. Examples of MPS flow results are shown in Figure 6.

Notwithstanding this early enthusiasm about SPECT MPI flow, there are well-known limitations of this use with ${ }^{99 \mathrm{~m}} \mathrm{Tc}$-sestamibi or tetrofosmin. Notably, the myocardial extraction of these radiotracers from the blood is substantially reduced at high flow rates, 


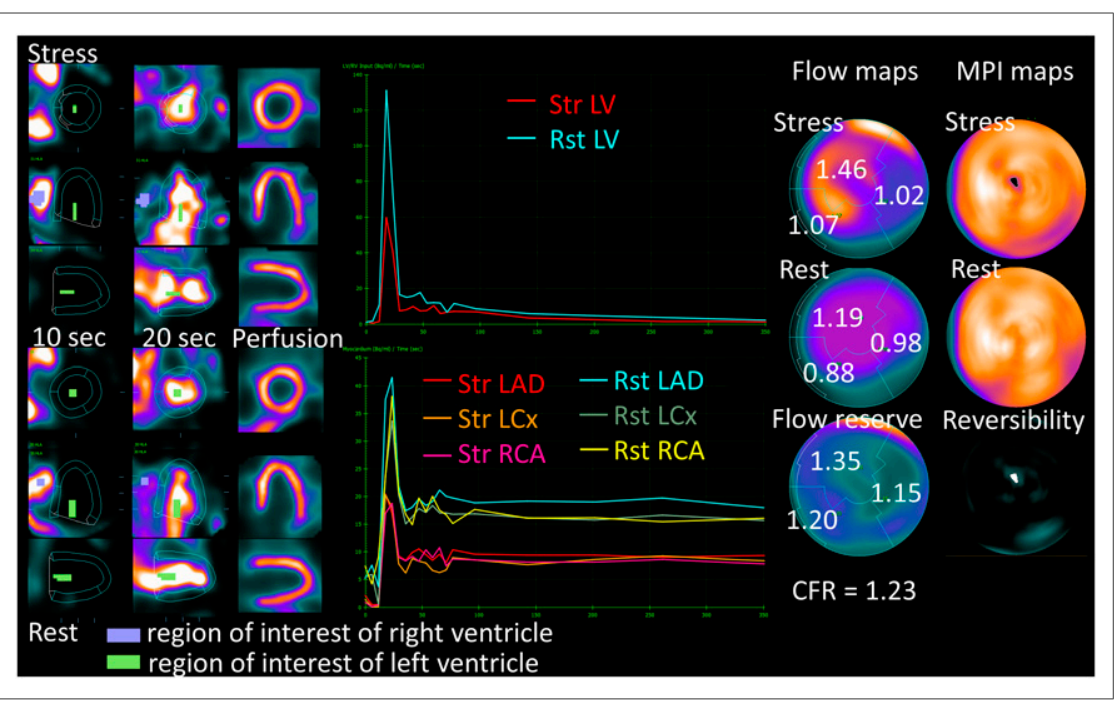

FIGURE 6. SPECT MBF information. One-day ${ }^{99 m T c}$ sestamibi stress/rest protocol $(259 \mathrm{MBq}$ for stress and $777 \mathrm{MBq}$ for rest) was used. Stress and rest flows are computed from early dynamic imaging for each vascular territory, by deriving regional time-activity curves for myocardium and for input blood in left atrium or left ventricle and applying compartmental models. These values can be used to calculate MFR as ratio between stress and rest MBF. Reduced MFR $(<2.0)$ may be useful to identify presence of multivessel disease leading to balanced ischemia. This patient had normal regional myocardial perfusion but decreased MFR and was ultimately found to have obstructive triple-vessel disease on coronary angiography. $L A D=$ left anterior descending; $L C x=$ left circumflex; LV = left ventricle; RCA = right coronary artery; Rst = rest; Str = stress. (Courtesy of Dr. Alejandro Meretta, Instituto Cardiovascular de Buenos Aires.)
However, SPECT-derived MFR was similar to PET values and had high diagnostic accuracy for PET-defined ischemia $(>93 \%)$ or ischemia defined by invasive fractional flow reserve $(>81 \%)$ (58). Therefore, although SPECT flow measurements may not be as robust as PET, they are emerging as an important ancillary measure. Notably, no additional tracer injection is needed, thus minimizing additional costs and adding no radiation. Clinical validation studies for MBF measurement with solid-state MPI are summarized in Table 1.

\section{EARLY LEFT VENTRICULAR EJECTION FRACTION}

SPECT measurements of MBF and MFR require an acquisition at the time of radiotracer injection. This facilitates measurement of stress left ventricular ejection fraction (LVEF) and LVEF reserve (defined as stress LVEF - rest LVEF)—which are important ancillary PET measurements. Although poststress LVEF changes are useful with SPECT, they are obtained at least $15 \mathrm{~min}$ after stress and are less sensitive than measurements during stress. High LVEF reserve has been shown to significantly reduce the probability of severe corowhich requires mathematic compensation. However, even after compensation, this roll-off phenomenon increases result variability. Despite these concerns, good agreement between PET and SPECT flows has been shown in head-to-head studies $(58,62)$. In a study of 28 consecutive patients undergoing SPECT with AC and ${ }^{13} \mathrm{~N}$-ammonia PET, $K_{1}$ and MFR were computed without using any extraction

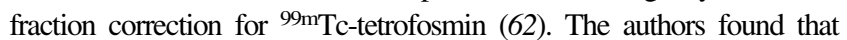
the hyperemic SPECT MBF values were lower than expected and, consequently, that MFR was lower than PET values (62). Agostini et al. compared ${ }^{99} \mathrm{~m}$ Tc-sestamibi SPECT-derived MBF and MFR, using a solid-state camera and a correction for ${ }^{99 \mathrm{~m}} \mathrm{Tc}$ extraction fraction, with values obtained with ${ }^{15} \mathrm{O}$-water PET in 30 patients (58). SPECT MBF measurements were lower than PET values on stress and rest acquisitions. nary artery disease during dipyridamole stress ${ }^{82} \mathrm{Rb}$ PET (64). The feasibility of LVEF reserve measurements using early poststress acquisitions with a dedicated cardiac solid-state SPECT scanner has been demonstrated in a study of 50 patients (65). Significantly lower LVEF reserve was obtained in the fifth and the ninth minutes after regadenoson bolus in patients with significant ischemia compared with patients without ischemia. In the future, it may be feasible to measure LVEF reserve and MBF from the same early SPECT acquisitions, providing critical ancillary diagnostic and prognostic markers.

\section{LARGE-SCALE CLINICAL VALIDATION}

The increasing clinical use of solid-state SPECT camera systems has led to a large body of evidence validating their prognostic and

TABLE 1

Human Validation Studies of MBF Quantification with Solid-State SPECT Cameras

\begin{tabular}{|c|c|c|c|c|c|}
\hline Study & Cases $(n)$ & System & Tracer & Validated with... & Model used \\
\hline Ben-Haim (57) & 95 & D-SPECT & Sestamibi & Perfusion angiography & Factor analysis \\
\hline Ben Bouallègue (63) & 23 & Discovery $530 \mathrm{c}$ & Tetrofosmin & iFFR & $1 \mathrm{TC}$ \\
\hline Nkoulou (62) & 28 & Discovery $570 \mathrm{c}$ & Tetrofosmin & ${ }^{13} \mathrm{~N}$-ammonia & $1 \mathrm{TC}$ \\
\hline Miyagawa (73) & 69 & Discovery $530 \mathrm{c}$ & Tetrofosmin/sestamibi & iFFR & $1 \mathrm{TC}$ \\
\hline Wells (59) & 31 & Discovery $530 \mathrm{c}$ & Tetrofosmin & ${ }^{82} \mathrm{Rb}$ or ${ }^{13} \mathrm{~N}$-ammonia & $1 \mathrm{TC}$ \\
\hline Agostini (58) & 30 & D-SPECT & Sestamibi & ${ }^{15} \mathrm{O}$-water iFFR & Net retention model \\
\hline $\operatorname{Han}(60)$ & 34 & Discovery $530 \mathrm{c}$ & Thallium/tetrofosmin & iFFR & $1 \mathrm{TC}$ \\
\hline $\mathrm{Ma}(61)$ & 40 & Discovery $530 \mathrm{c}$ & Sestamibi & RRG vs. solid-state & $1 \mathrm{TC}$ \\
\hline Zavadovsky (74) & 23 & Discovery $570 c$ & Sestamibi & iFFR & Net retention model \\
\hline
\end{tabular}

$\mathrm{iFFR}=$ invasive fractional flow reserve; 1TC = 1-tissue-compartment model; RRG = rapid rotating gantry. 
TABLE 2

Major Diagnostic $(n>100)$ and Prognostic $(n>1,000)$ Validation Studies for Solid-State SPECT MPI

\begin{tabular}{|c|c|c|c|c|c|c|}
\hline Study & Cases $(n)$ & System & Comparator & Tracer & Dose & Stress/rest time (min) \\
\hline \multicolumn{7}{|c|}{ Diagnostic validation } \\
\hline Nakazato (20) & 56 ICA, 86 LLK & D-SPECT & ICA & Sestamibi, dual-isotope & $\mathrm{SD}$ & $2 / 4$ \\
\hline Gimelli (15) & 137 & Discovery $530 c$ & ICA & Tetrofosmin & LD & $7 / 6$ \\
\hline Duvall (22) & 160 & Discovery 530c & ICA & Sestamibi & LD, SD & $3-5 / 3-5$ \\
\hline Mouden (75) & 100 & Discovery $570 c$ & iFFR & Tetrofosmin & $\mathrm{SD}$ & $5 / 4$ \\
\hline Nakazato (21) & 67 ICA, 51 LLK & D-SPECT & ICA & Sestamibi, dual-isotope & SD & $2-6 / 6-12$ \\
\hline Chikamori (76) & 102 & Discovery 530c & ICA/iFFR & $\begin{array}{l}\text { Tetrofosmin, } \\
\text { sestamibi }\end{array}$ & LD & $10 / 6$ \\
\hline Sharir (25) & 208 ICA, 76 LLk & Discovery 530c & ICA & Sestamibi & LD vs. SD & LD: 5-7/5-7; SD: 5/3 \\
\hline Betancur (45) & 1,160 & D-SPECT & ICA & Sestamibi & LD, SD & $4-6 / 6-10$ \\
\hline Betancur (68) & 1,638 & $\begin{array}{l}\text { D-SPECT, Discovery } \\
530 \text { c or } 570 c\end{array}$ & ICA & $\begin{array}{l}\text { Sestamibi, tetrofosmin, } \\
\text { dual-isotope }\end{array}$ & LD, SD & $4-6 / 6-10$ \\
\hline Gimelli (77) & 1,161 & Discovery $530 c$ & ICA & Tetrofosmin & LD & $7 / 6$ \\
\hline \multicolumn{7}{|c|}{ Prognostic validation } \\
\hline Nakazato (78) & 1,613 & D-SPECT & All-cause mortality & $\begin{array}{l}\text { Sestamibi, } \\
\text { dual-isotope }\end{array}$ & SD & $\begin{array}{l}\text { Sestamibi: } 2 / 4 \\
\text { dual: } 6 / 4\end{array}$ \\
\hline Chowdhury (79) & 1,109 (165 with ICA) & Discovery 530c & MACE, ICA & Tetrofosmin & $\mathrm{SD}$ & $3-6 / 3-6$ \\
\hline De Lorenzo (80) & 1,396 & Discovery 530c & All-cause mortality & Sestamibi & SD & $4 / 8$ \\
\hline Yokota (81) & $\begin{array}{l}\text { 1,288 solid-state, } \\
362 \text { conventional }\end{array}$ & Discovery 570c & MACE & Tetrofosmin & LD & $5 /-$ \\
\hline Engbers (82) & 4,057 & Discovery 570c & MACE & Tetrofosmin & $\mathrm{SD}$ & $5 / 4$ \\
\hline Lima (83) & 3,554 & Discovery 530c & MACE & Sestamibi & LD & $3 / 6$ \\
\hline Lima (84) & 2,930 & Discovery 530c & MACE & Sestamibi & SD, LD & $3 / 6$ \\
\hline Betancur (85) & 2,619 & D-SPECT & MACE & Sestamibi & $\mathrm{SD}$ & $4-6 / 6-10$ \\
\hline van Dijk (86) & 1,255 & Discovery $570 c$ & MACE & Tetrofosmin & SD vs. LD & SD: 5/4; LD: $8 / 6$ \\
\hline Otaki (67) & 19,495 & $\begin{array}{l}\text { D-SPECT, } \\
\text { Discovery 530c }\end{array}$ & MACE & $\begin{array}{l}\text { Tetrofosmin, } \\
\text { sestamibi, } \\
\text { dual-isotope }\end{array}$ & LD, SD & $4-6 / 6-10$ \\
\hline
\end{tabular}

ICA = invasive coronary angiography; LLk = low likelihood of coronary artery disease; SD = standard dose; LD = low-dose; iFFR = invasive fractional flow reserve; MACE = major adverse cardiac events.

Overall effective dose of test is LD if dose is $\leq 6 \mathrm{mSv}$; SD if above $6 \mathrm{mSv}$. Acquisition time is for default view if more than 1 position is taken.

diagnostic utility. Major studies of the diagnostic or prognostic utility of solid-state SPECT cameras are outlined in Table 2. One of the largest such efforts is a large, multicenter, international

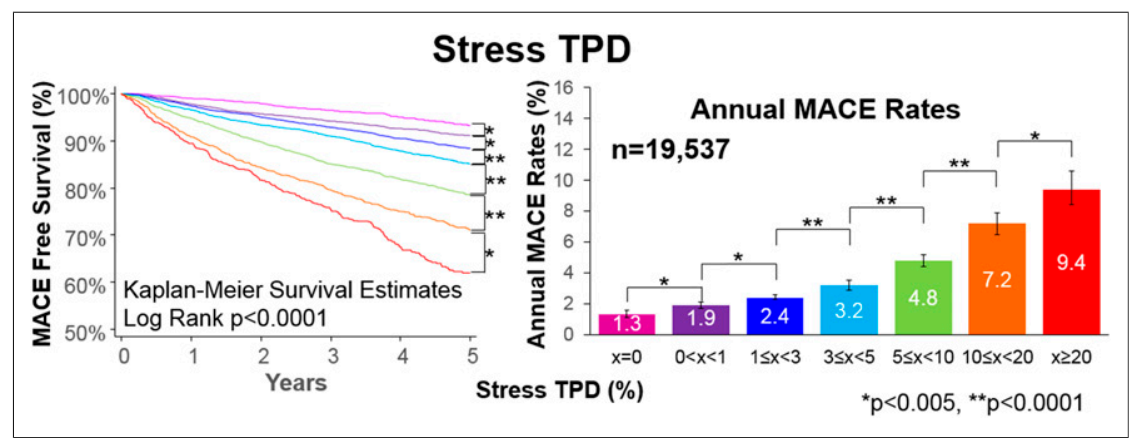

FIGURE 7. Prognostic value of quantitative perfusion analysis from REFINE SPECT with solidstate MPI. Quantitative perfusion assessments with TPD provide fine gradation of cardiovascular risk. Each increase in TPD extent, including $<1 \%$ ischemia compared with no ischemia, was associated with significant increase in combined outcome of death, nonfatal myocardial infarction, unstable angina, or late revascularization. MACE $=$ major adverse cardiovascular events. (Reprinted with permission of (67).) registry - the Registry of Fast Myocardial Perfusion Imaging with Next Generation SPECT (REFINE SPECT) (66). The novel collaborative design of this registry features the contribution of clinical data and image datasets from 9 (to date) investigative centers around the world into a comprehensive clinicalimaging database established at the central core laboratory at Cedars-Sinai Medical Center with centralized automatic quantitative analysis (66). To date, 20,418 solidstate MPI scans with collected prognostic data have been included in the registry. Initial results from the registry have shown that quantitative perfusion analysis, measured as total perfusion deficit (TPD), provides fine gradation of risk (67). Increasing TPD, even at very low levels, is associated with increasing risk of death, nonfatal myocardial infarction, unstable angina, or late revascularization (67). Notably, patients with more than 0 but less than $1 \%$ TPD had a $40 \%$ higher risk of events than patients 
with a TPD of 0 , demonstrating the importance of even small deficits, which may be missed without the higher spatial resolution of new solid-state SPECT cameras (Fig. 7) (67).

The registry has also allowed researchers to perform various collaborative projects, including the development of novel machine learning methods tailored for the analysis of solid-state SPECT MPI and subsequent integration of quantitative image data variables with prescan clinical information. A machine-learning score derived with deep-convolutional neural networks has been shown to have superior diagnostic accuracy for obstructive coronary artery disease compared with quantitative ischemia assessments $(45,68)$. A separate machine-learning algorithm had superior prognostic accuracy compared with either quantitative ischemia assessment or physician assessment (45). Other validation studies have been performed for solid-state MPI; these include validation of diagnostic accuracy including 2-position imaging and AC (21), determination of the transient ischemic dilation limits (69), and prognostic performance (67). Given the rich data collected, further validation studies are expected from this large multicenter cohort in the future.

\section{FUTURE HARDWARE DESIGNS}

Although these recent SPECT hardware improvements have proven to be clinically important, further advancements are still possible. One possible area for improvement is additional gains in photon sensitivity. Although more sensitive than conventional Anger cameras, even dedicated cardiac SPECT scanners detect less than $0.1 \%$ of emitted photons. Preclinical studies have demonstrated that higher efficiency may be possible. In a simulation, Zeng et al. (70) demonstrated that multidivergent beam collimators could double the photon sensitivity of current SPECT camera systems without sacrificing spatial resolution. Simulations of multipinhole designs with paired curved paraboloid or hemi-ellipsoid detectors could improve photon sensitivity by up to $300 \%(71,72)$. In phantom studies, these designs have improved photon sensitivity and spatial resolution compared with published characteristics or commercially available solid-state camera systems (72). Nevertheless, these published reports are still preclinical, and it remains to be seen if these designs will be cost-effective and adequate for clinical implementation.

\section{SUMMARY}

Solid-state cardiac SPECT offers substantial improvements in photon sensitivity and spatial resolution over conventional Anger cameras. These advances are due to novel collimator and geometric designs as well as incorporation of CZT crystals. Solidstate camera systems have facilitated dramatic reductions in both imaging time and radiation dose while maintaining high diagnostic accuracy. Growing clinical experience has validated the excellent diagnostic and prognostic utility attained with these camera systems. With the CZT systems, simultaneous dual-isotope SPECT is feasible and has been shown to be useful in selected applications. The improved technical performance has also facilitated measurement of ancillary high-risk markers including MBF and LVEF reserve. Although these advances have already provided a substantial improvement over the use of standard $\mathrm{NaI}$ crystal-based systems, the full clinical potential of solid-state cardiac SPECT camera systems has not yet been realized.

\section{DISCLOSURE}

No potential conflict of interest relevant to this article was reported.

\section{REFERENCES}

1. Slomka PJ, Patton JA, Berman DS, Germano G. Advances in technical aspects of myocardial perfusion SPECT imaging. J Nucl Cardiol. 2009;16:255-276.

2. Sharir T, Ben-Haim S, Merzon K, et al. High-speed myocardial perfusion imaging: initial clinical comparison with conventional dual detector anger camera imaging. JACC Cardiovasc Imaging. 2008;1:156-163.

3. Imbert L, Poussier S, Franken PR, et al. Compared performance of high-sensitivity cameras dedicated to myocardial perfusion SPECT: a comprehensive analysis of phantom and human images. J Nucl Med. 2012;53:1897-1903.

4. Volokh L, Hugg J, Blevis I, Asma E, Jansen F, Manjeshwar R. Effect of detector energy response on image quality of myocardial perfusion SPECT. Nuclear Science Symposium Conference Record, 2008 NSS'08 IEEE. 2008:4043-4046.

5. Siman W, Kappadath SC. Performance characteristics of a new pixelated portable gamma camera. Med Phys. 2012;39:3435-3444.

6. Gunter DL. Gamma camera collimator characteristics and design. In: Henkin RE, ed. Nuclear Medicine. Vol 1. Philadelphia, PA: Elsevier; 2006:107-126.

7. Goshen E, Beilin L, Stern E, Kenig T, Goldkorn R, Ben-Haim S. Feasibility study of a novel general purpose CZT-based digital SPECT camera: initial clinical results. EJNMMI Phys. 2018;5:6.

8. Keidar Z, Raysberg I, Lugassi R, Frenkel A, Israel O. Novel cadmium zinc telluride based detector general purpose gamma camera: initial evaluation and comparison with a standard camera [abstract]. J Nucl Med. 2016;57(suppl):259.

9. Schepis T, Gaemperli O, Koepfli P, et al. Use of coronary calcium score scans from stand-alone multislice computed tomography for attenuation correction of myocardial perfusion SPECT. Eur J Nucl Med Mol Imaging. 2007;34:11-19.

10. Grossmann M, Giannopoulos AA, Bechtiger FA, et al. Ultra-low-dose computed tomography for attenuation correction of cadmium-zinc-telluride single photon emission computed tomography myocardial perfusion imaging. J Nucl Cardiol. June 19, 2018 [Epub ahead of print].

11. Caobelli F, Akin M, Thackeray JT, et al. Diagnostic accuracy of cadmium-zinctelluride-based myocardial perfusion SPECT: impact of attenuation correction using a co-registered external computed tomography. Eur Heart J Cardiovasc Imaging. 2016;17:1036-1043.

12. Buechel RR, Herzog BA, Husmann L, et al. Ultrafast nuclear myocardial perfusion imaging on a new gamma camera with semiconductor detector technique: first clinical validation. Eur J Nucl Med Mol Imaging. 2010;37:773-778.

13. Sharir T, Slomka PJ, Hayes SW, et al. Multicenter trial of high-speed versus conventional single-photon emission computed tomography imaging: quantitative results of myocardial perfusion and left ventricular function. J Am Coll Cardiol. 2010;55:1965-1974.

14. Henzlova MJ, Duvall W. The future of SPECT MPI: time and dose reduction. J Nucl Cardiol. 2011;18:580-587.

15. Gimelli A, Bottai M, Genovesi D, Giorgetti A, Di Martino F, Marzullo P. High diagnostic accuracy of low-dose gated-SPECT with solid-state ultrafast detectors: preliminary clinical results. Eur J Nucl Med Mol Imaging. 2012;39:83-90.

16. Nkoulou R, Pazhenkottil AP, Kuest SM, et al. Semiconductor detectors allow low-dose-low-dose 1-day SPECT myocardial perfusion imaging. J Nucl Med. 2011;52:1204-1209.

17. Bateman T, McGhie A, Courter S, Burgett E, Cullom S, Case J. Prospective study of ultra-low dose stress-only solid-state SPECT: comparison of efficiency, dosimetry and outcomes versus traditional-dose attenuation-corrected stress-only Anger SPECT [abstract]. J Am Coll Cardiol. 2012;59(suppl):E1316.

18. Nakazato R, Berman DS, Hayes SW, et al. Myocardial perfusion imaging with a solid-state camera: simulation of a very low dose imaging protocol. J Nucl Med. 2013;54:373-379.

19. Schauer DA, Linton OW. NCRP Report No. 160, Ionizing Radiation Exposure of the Population of the United States, medical exposure-are we doing less with more, and is there a role for health physicists? Health Phys. 2009;97:1-5.

20. Nakazato R, Tamarappoo BK, Kang X, et al. Quantitative upright-supine highspeed SPECT myocardial perfusion imaging for detection of coronary artery disease: correlation with invasive coronary angiography. J Nucl Med. 2010; 51:1724-1731.

21. Nakazato R, Slomka PJ, Fish M, et al. Quantitative high-efficiency cadmiumzinc-telluride SPECT with dedicated parallel-hole collimation system in obese patients: results of a multi-center study. J Nucl Cardiol. 2015;22:266-275.

22. Duvall WL, Slomka PJ, Gerlach JR, et al. High-efficiency SPECT MPI: comparison of automated quantification, visual interpretation, and coronary angiography. J Nucl Cardiol. 2013;20:763-773.

23. Einstein AJ, Blankstein R, Andrews H, et al. Comparison of image quality, myocardial perfusion, and left ventricular function between standard imaging and single-injection ultra-low-dose imaging using a high-efficiency SPECT camera: the MILLISIEVERT study. J Nucl Med. 2014;55:1430-1437. 
24. Einstein AJ, Johnson LL, DeLuca AJ, et al. Radiation dose and prognosis of ultra-low-dose stress-first myocardial perfusion SPECT in patients with chest pain using a high-efficiency camera. J Nucl Med. 2015;56:545-551.

25. Sharir T, Pinskiy M, Pardes A, et al. Comparison of the diagnostic accuracies of very low stress-dose with standard-dose myocardial perfusion imaging: automated quantification of one-day, stress-first SPECT using a CZT camera. $\mathrm{J} \mathrm{Nucl}$ Cardiol. 2016;23:11-20.

26. Nkoulou R, Pazhenkottil AP, Wolfrum M, et al. High efficiency gamma camera enables ultra-low fixed dose stress/rest myocardial perfusion imaging. Eur Heart J Cardiovasc Imaging. 2019;20:218-224.

27. Palyo RJ, Sinusas AJ, Liu YH. High-sensitivity and high-resolution SPECT/CT systems provide substantial dose reduction without compromising quantitative precision for assessment of myocardial perfusion and function. J Nucl Med. 2016;57:893-899.

28. Slomka PJ, Rubeaux M, Germano G. Quantification with normal limits: new cameras and low-dose imaging. J Nucl Cardiol. 2017;24:1637-1640.

29. Chang SM, Nabi F, Xu J, Raza U, Mahmarian JJ. Normal stress-only versus standard stress/rest myocardial perfusion imaging: similar patient mortality with reduced radiation exposure. J Am Coll Cardiol. 2010;55:221-230.

30. Duvall WL, Guma KA, Kamen J, et al. Reduction in occupational and patient radiation exposure from myocardial perfusion imaging: impact of stress-only imaging and high-efficiency SPECT camera technology. J Nucl Med. 2013;54:1251-1257.

31. Rozanski A, Gransar H, Hayes SW, et al. Temporal trends in the frequency of inducible myocardial ischemia during cardiac stress testing: 1991 to 2009. J Am Coll Cardiol. 2013;61:1054-1065.

32. Kacperski K, Erlandsson K, Ben-Haim S, Van Gramberg D, Hutton BF. Iterative deconvolution of simultaneous dual radionuclide projections for CdZnTe based cardiac SPECT. IEEE Nuclear Science Symposium Conference Record, 2008. NSS'08. 2008:5260-5263.

33. Kiat H, Germano G, Friedman J, et al. Comparative feasibility of separate or simultaneous rest thallium-201/stress technetium-99m-sestamibi dual-isotope myocardial perfusion SPECT. J Nucl Med. 1994;35:542-548.

34. Steele PP, Kirch DL, Koss JE. Comparison of simultaneous dual-isotope multipinhole SPECT with rotational SPECT in a group of patients with coronary artery disease. J Nucl Med. 2008;49:1080-1089.

35. Ben-Haim S, Kacperski K, Hain S, et al. Simultaneous dual-radionuclide myocardial perfusion imaging with a solid-state dedicated cardiac camera. Eur J Nucl Med Mol Imaging. 2010;37:1710-1721.

36. Fan P, Hutton BF, Holstensson M, et al. Scatter and crosstalk corrections for ${ }^{99 \mathrm{~m}} \mathrm{Tc} /{ }^{123} \mathrm{I}$ dual-radionuclide imaging using a CZT SPECT system with pinhole collimators. Med Phys. 2015;42:6895-6911.

37. Tamarappoo B, Otaki Y, Manabe O, et al. Simultaneous Tc-99m PYP/Tl-201 dual-isotope SPECT myocardial imaging in patients with suspected cardiac amyloidosis. J Nucl Cardiol. June 6, 2019 [Epub ahead of print].

38. Matsuo S, Nakajima K, Kinuya S, Yamagishi M. Diagnostic utility of ${ }^{123}$ IBMIPP imaging in patients with Takotsubo cardiomyopathy. J Cardiol. 2014;64:49-56.

39. Yamada Y, Nakano S, Gatate Y, et al. Feasibility of simultaneous 99mTc-tetrofosmin and 123I-BMIPP dual-tracer imaging with cadmium-zinc-telluride detectors in patients undergoing primary coronary intervention for acute myocardial infarction. J Nucl Cardiol. January 2, 2019 [Epub ahead of print].

40. Blaire T, Bailliez A, Ben Bouallegue F, Bellevre D, Agostini D, Manrique A. Determination of the heart-to-mediastinum ratio of ${ }^{123}$ I-MIBG uptake using dual-isotope $\left({ }^{123} \mathrm{I}-\mathrm{MIBG} /{ }^{99 \mathrm{~m}} \mathrm{Tc}\right.$-tetrofosmin) multipinhole cadmium-zinc-telluride SPECT in patients with heart failure. J Nucl Med. 2018;59:251-258.

41. D'estanque E, Hedon C, Lattuca B, et al. Optimization of a simultaneous dualisotope ${ }^{201} \mathrm{Tl} /{ }^{123} \mathrm{I}-\mathrm{MIBG}$ myocardial SPECT imaging protocol with a CZT camera for trigger zone assessment after myocardial infarction for routine clinical settings: are delayed acquisition and scatter correction necessary? J Nucl Cardiol. 2017;24:1361-1369.

42. Blaire T, Bailliez A, Ben Bouallegue F, Bellevre D, Agostini D, Manrique A. First assessment of simultaneous dual isotope $\left({ }^{123} \mathrm{I} /{ }^{99 \mathrm{~m}} \mathrm{Tc}\right)$ cardiac SPECT on two different CZT cameras: a phantom study. J Nucl Cardiol. 2018;25:1692-1704.

43. Nishiyama Y, Miyagawa M, Kawaguchi N, et al. Combined supine and prone myocardial perfusion single-photon emission computed tomography with a cadmium zinc telluride camera for detection of coronary artery disease. Circ J. 2014;78:1169-1175.

44. Fiechter M, Gebhard C, Fuchs TA, et al. Cadmium-zinc-telluride myocardial perfusion imaging in obese patients. J Nucl Med. 2012;53:1401-1406.

45. Betancur J, Hu LH, Commandeur F, et al. Deep learning analysis of uprightsupine high-efficiency SPECT myocardial perfusion imaging for prediction of obstructive coronary artery disease: a multicenter study. J Nucl Med. 2019;60:664-670.
46. Daou D, Sabbah R, Coaguila C, Boulahdour H. Feasibility of data-driven cardiac respiratory motion correction of myocardial perfusion CZT SPECT: a pilot study. J Nucl Cardiol. 2017;24:1598-1607.

47. Buechel RR, Husmann L, Pazhenkottil AP, et al. Myocardial perfusion imaging with real-time respiratory triggering: impact of inspiration breath-hold on left ventricular functional parameters. J Nucl Cardiol. 2010;17:848-852.

48. Chan C, Harris M, Le M, et al. End-expiration respiratory gating for a highresolution stationary cardiac SPECT system. Phys Med Biol. 2014;59:62676287.

49. Clerc OF, Fuchs TA, Possner M, et al. Real-time respiratory triggered SPECT myocardial perfusion imaging using CZT technology: impact of respiratory phase matching between SPECT and low-dose CT for attenuation correction. Eur Heart J Cardiovasc Imaging. 2017;18:31-38.

50. Kennedy JA, William Strauss H. Motion detection and amelioration in a dedicated cardiac solid-state CZT SPECT device. Med Biol Eng Comput. 2017;55:663-671.

51. Kennedy JA, Israel O, Frenkel A. 3D iteratively reconstructed spatial resolution map and sensitivity characterization of a dedicated cardiac SPECT camera. J Nucl Cardiol. 2014;21:443-452.

52. Oddstig J, Martinsson E, Jögi J, Engblom H, Hindorf C. Differences in attenuation pattern in myocardial SPECT between CZT and conventional gamma cameras. J Nucl Cardiol. May 23, 2018 [Epub ahead of print].

53. Tsuboi K, Nagaki A, Shibutani T, Onoguchi M. Optimal choice of OSEM and SD reconstruction algorithms in CZT SPECT for hypertrophic cardiomyopathy patients. J Nucl Cardiol. March 7, 2019 [Epub ahead of print].

54. Fiechter M, Ghadri JR, Gebhard C, et al. Diagnostic value of ${ }^{13} \mathrm{~N}$-ammonia myocardial perfusion PET: added value of myocardial flow reserve. $J \mathrm{Nucl}$ Med. 2012;53:1230-1234.

55. Ziadi MC, Dekemp RA, Williams KA, et al. Impaired myocardial flow reserve on rubidium-82 positron emission tomography imaging predicts adverse outcomes in patients assessed for myocardial ischemia. J Am Coll Cardiol. 2011;58:740-748.

56. Murthy VL, Naya M, Foster CR, et al. Improved cardiac risk assessment with noninvasive measures of coronary flow reserve. Circulation. 2011;124:22152224.

57. Ben-Haim S, Murthy VL, Breault C, et al. Quantification of myocardial perfusion reserve using dynamic SPECT imaging in humans: a feasibility study. J Nucl Med. 2013;54:873-879.

58. Agostini D, Roule V, Nganoa C, et al. First validation of myocardial flow reserve assessed by dynamic ${ }^{99 \mathrm{~m}}$ Tc-sestamibi CZT-SPECT camera: head to head comparison with ${ }^{15} \mathrm{O}$-water PET and fractional flow reserve in patients with suspected coronary artery disease. The WATERDAY study. Eur J Nucl Med Mol Imaging. 2018;45:1079-1090.

59. Wells RG, Marvin B, Poirier M, Renaud J, deKemp RA, Ruddy TD. Optimization of SPECT measurement of myocardial blood flow with corrections for attenuation, motion, and blood binding compared with PET. J Nucl Med. 2017;58:2013-2019.

60. Han S, Kim YH, Ahn JM, et al. Feasibility of dynamic stress ${ }^{201} \mathrm{Tl} / \mathrm{rest}^{99 \mathrm{~m}} \mathrm{Tc}$ tetrofosmin single photon emission computed tomography for quantification of myocardial perfusion reserve in patients with stable coronary artery disease. Eur J Nucl Med Mol Imaging. 2018;45:2173-2180.

61. Ma R, Wang L, Wu D, et al. Myocardial blood flow quantitation in patients with congestive heart failure: head-to-head comparison between rapid-rotating gantry SPECT and CZT SPECT. J Nucl Cardiol. February 19, 2019 [Epub ahead of print].

62. Nkoulou R, Fuchs TA, Pazhenkottil AP, et al. Absolute myocardial blood flow and flow reserve assessed by gated SPECT with cadmium-zinc-telluride detectors using ${ }^{99 \mathrm{~m}}$ Tc-tetrofosmin: head-to-head comparison with ${ }^{13} \mathrm{~N}$-ammonia PET. J Nucl Med. 2016;57:1887-1892.

63. Ben Bouallègue F, Roubille F, Lattuca B, et al. SPECT myocardial perfusion reserve in patients with multivessel coronary disease: correlation with angiographic findings and invasive fractional flow reserve measurements. $\mathrm{J} \mathrm{Nucl}$ Med. 2015;56:1712-1717.

64. Dorbala S, Vangala D, Sampson U, Limaye A, Kwong R, Di Carli MF. Value of vasodilator left ventricular ejection fraction reserve in evaluating the magnitude of myocardium at risk and the extent of angiographic coronary artery disease: a ${ }^{82} \mathrm{Rb}$ PET/CT study. J Nucl Med. 2007;48:349-358.

65. Brodov Y, Fish M, Rubeaux M, et al. Quantitation of left ventricular ejection fraction reserve from early gated regadenoson stress Tc-99m high-efficiency SPECT. J Nucl Cardiol. 2016;23:1251-1261.

66. Slomka PJ, Betancur J, Liang JX, et al. Rationale and design of the REgistry of Fast Myocardial Perfusion Imaging with NExt generation SPECT (REFINE SPECT). J Nucl Cardiol. June 19, 2018 [Epub ahead of print]. 
67. Otaki Y, Betancur J, Sharir T, et al. 5-year prognostic value of quantitative versus visual MPI in subtle perfusion defects: results from REFINE SPECT. JACC Cardiovasc Imaging. June 8, 2019 [Epub ahead of print].

68. Betancur J, Commandeur F, Motlagh M, et al. Deep learning for prediction of obstructive disease from fast myocardial perfusion SPECT: a multicenter study. JACC Cardiovasc Imaging. 2018;11:1654-1663.

69. Hu L, Betancur J, Sharir T, et al. The normal limits of transient ischemic dilatation ratio for different protocols on CZT cameras: a report from the International Multicenter Prognostic REFINE SPECT Registry. J Nucl Cardiol. 2019. In press.

70. Zeng GL, Stevens AM. Multidivergent-beam stationary cardiac SPECT. Med Phys. 2009;36:2860-2869.

71. Dey J. Improvement of performance of cardiac SPECT camera using curved detectors with pinholes. IEEE Trans Nucl Sci. 2012;59:334-347.

72. Bhusal N, Dey J, Xu J, et al. Performance analysis of a high-sensitivity multipinhole cardiac SPECT system with hemi-ellipsoid detectors. Med Phys. 2019;46:116-126.

73. Miyagawa M, Nishiyama Y, Uetani T, et al. Estimation of myocardial flow reserve utilizing an ultrafast cardiac SPECT: comparison with coronary angiography, fractional flow reserve, and the SYNTAX score. Int J Cardiol. 2017;244:347-353.

74. Zavadovsky KV, Mochula AV, Boshchenko AA, et al. Absolute myocardial blood flows derived by dynamic CZT scan vs invasive fractional flow reserve: correlation and accuracy. J Nucl Cardiol. March 7, 2019 [Epub ahead of print].

75. Mouden M, Ottervanger JP, Knollema S, et al. Myocardial perfusion imaging with a cadmium zinc telluride-based gamma camera versus invasive fractional flow reserve. Eur J Nucl Med Mol Imaging. 2014;41:956-962.

76. Chikamori T, Hida S, Tanaka N, et al. Diagnostic performance of a cadmium-zinc-telluride single-photon emission computed tomography system with low-dose technetium-99m as assessed by fractional flow reserve. Circ J. 2016;80:1217-1224.

77. Gimelli A, Pugliese NR, Kusch A, Giorgetti A, Marzullo P. Accuracy of cadmium-zinc-telluride imaging in detecting single and multivessel coronary artery disease: is there any gender difference? Int J Cardiol. 2019;274:388-393.
78. Nakazato R, Berman DS, Gransar H, et al. Prognostic value of quantitative highspeed myocardial perfusion imaging. J Nucl Cardiol. 2012;19:1113-1123.

79. Chowdhury FU, Vaidyanathan S, Bould M, et al. Rapid-acquisition myocardial perfusion scintigraphy (MPS) on a novel gamma camera using multipinhole collimation and miniaturized cadmium-zinc-telluride (CZT) detectors: prognostic value and diagnostic accuracy in a 'real-world' nuclear cardiology service. Eur Heart J Cardiovasc Imaging. 2014;15:275-283.

80. De Lorenzo A, Peclat T, Amaral AC, Lima RSL. Prognostic evaluation in obese patients using a dedicated multipinhole cadmium-zinc telluride SPECT camera. Int J Cardiovasc Imaging. 2016;32:355-361.

81. Yokota S, Mouden M, Ottervanger JP, et al. Prognostic value of normal stress-only myocardial perfusion imaging: a comparison between conventional and CZT-based SPECT. Eur J Nucl Med Mol Imaging. 2016;43: 296-301.

82. Engbers EM, Timmer JR, Mouden M, Knollema S, Jager PL, Ottervanger JP. Prognostic value of myocardial perfusion imaging with a cadmium-zinc-telluride SPECT camera in patients suspected of having coronary artery disease. $J \mathrm{Nucl}$ Med. 2017;58:1459-1463.

83. Lima R, Peclat T, Soares T, Ferreira C, Souza AC, Camargo G. Comparison of the prognostic value of myocardial perfusion imaging using a CZTSPECT camera with a conventional Anger camera. J Nucl Cardiol. 2017;24: 245-251.

84. Lima RSL, Peclat TR, Souza A, et al. Prognostic value of a faster, low-radiation myocardial perfusion SPECT protocol in a CZT camera. Int $J$ Cardiovasc Imaging. 2017;33:2049-2056.

85. Betancur J, Otaki Y, Motwani M, et al. Prognostic value of combined clinical and myocardial perfusion imaging data using machine learning. JACC Cardiovasc Imaging. 2018;11:1000-1009.

86. van Dijk JD, Borren NM, Mouden M, van Dalen JA, Ottervanger JP, Jager PL. Effect of a patient-specific minimum activity in stress myocardial perfusion imaging using CZT-SPECT: prognostic value, radiation dose, and scan outcome. J Nucl Cardiol. 2018;25:26-35. 